\title{
Controlled Release of Doxorubicin from Electrospun Gelatin Nanofibers
}

\author{
Derya Mete ${ }^{1}$, Nesrin Horzum², Gülşah Şanlı Mohamed ${ }^{3}$ \\ ${ }^{1,3}$ Department of Chemistry, İzmir Institute of Technology, İzmir, Turkey \\ ${ }^{2}$ Department of Engineering Sciences, İzmir Katip Çelebi University, İzmir, Turkey \\ deryabostanbas@iyte.edu.tr
}

\section{Extended Abstract}

Electrospinning has been recognized as an effective and inexpensive technique for fabrication of long fibers from various materials including polymers, composites and biomacromolecules with diameters ranging from a few nanometers to few micrometers [1]. The electrospun fibers form a unique structure which have a very large surface area-to-volume ratio and high porosity with very small pore size. Therefore, electrospun fibers could be a very promising material for many biomedical applications such as drug delivery, wound dressing, artificial organ and medical prosthesis $[2,3]$.

Polymer-based drug delivery systems are used to improve the therapeutic properties of drugs in a safer, effective and reliable manner [4]. The variety of biodegradable polymers can be electrospinnable [5]. At present, researches on biodegradable nanofibers focus on development for delivering drugs and releasing them continuously over a period of time. Drug delivery with polymeric nanofibers with higher drug encapsulation efficiency and better stability than other drug formulations possess high surface-to-volume ratio which would accelerate the solubility of drug in the aqueous solution and enhance the efficiency of the drug $[6,7]$.

Gelatin is a natural biopolymer derived from animal collagen, having a long history of safe use in pharmaceuticals, cosmetics as well as food products [8]. Because of its great biocompatibility and biodegradability properties, gelatin has a broad applications in biomedical fields, especially as a drug delivery carrier [9].

In the current work electrospun fibers were developed as a new system for the delivery and release of an anticancer agent doxorubicin via electrospinning technique. The morphology of the fibers was analyzed by scanning electron microscopy(SEM), fourier transform infrared spectroscopy (FTIR). The fibers were made from gelatin as a biodegradable polymer and the release of doxorubicin was followed by UV-vis spectroscopy in phosphate buffer of $\mathrm{pH} 7.4$ at $25^{\circ} \mathrm{C}$ and $37^{\circ} \mathrm{C}$. The release profiles from gelatin electrospun fiber mats were compared with casting films with the same composition.

\section{References}

[1] A. Greiner and J. H. Wendorff, Angewandte Chemie. International Ed., vol. 46, pp. 5670-5703, 2007.

[2] H. Yoshimoto, Y. M. Shin, H. Terai, and J. P. Vacanti, Biomaterials, vol. 24, pp. 2077-2082, 2003.

[3] J. Zeng, X. Xu, X. Chen, Q. Liang, X. Bian, L. Yang, and X. Jing, Journal of controlled release: official journal of the Controlled Release Society, vol. 92, 227-231, 2003.

[4] R. Langer, Accounts of Chemical Research, vol. 26, pp. 537-542, 1993.

[5] B. Duan, X. Yuan, Y. Zhu, Y. Zhang, X. Li, Y. Zhang, and K. Yao, European Polymer Journal, vol. 42, pp. 2013-2022, 2006.

[6] J. Zeng, X. Xu, X. Chen, Q. Liang, X. Bian, L. Yang, and X. Jing, Journal of Controlled Release, vol. 92, pp. 227-231, 2003.

[7] Y. J. Son, W. J. Kim, and H. S. Yoo, Archives of pharmacal research, vol. 37, pp. 69-78, 2014.

[8] B. Idson, E. Braswell, "Gelatin," in Advances in Food Research, E.M. Mrak, G.F. Stewart Eds. Academic Press, 1957, pp. 235-338.

[9] Y. Zhang, H. Ouyang, C. T. Lim, S. Ramakrishna, and Z. M. Huang, Journal of biomedical materials research. Part B, Applied biomaterials, vol. 72, 156-165, 2005. 